\title{
Human Placenta-derived Stem Cell Transplantation
}

National Cancer Institute

\section{Source}

National Cancer Institute. Human Placenta-derived Stem Cell Transplantation. NCI

Thesaurus. Code C129407.

An adjunctive therapy to umbilical cord blood (UCB) transplantation that is used to augment stem cell engraftment in hematopoietic reconstitution. The process to extract human placenta-derived stem cells (HPDSCs) yields an enriched population of CD34positive hematopoietic stem cells that, when transplanted in conjunction with UCB stem cells, may provide a novel source for natural killer (NK) cells. 